\title{
Progressive waves of permanent form in continuously stratified fluids
}

\author{
Chia-Shun Yih \\ Department of Applied Mechanics and Engineering Science, The University of Michigan, Ann Arbor, Michigan 48104
}

(Received 21 February 1974)

\begin{abstract}
A theory for internal progressive waves of permanent form in any continuously stratified fluid is presented, and a calculation for the flow and the wave velocity is carried out for an exponential stratified fluid. The most important conclusion from this calculation, also valid for other weak stratifications, is that the wave velocity always decreases with the amplitude, provided the density gradient is weak and the wavelength is not too short. This conclusion is significant because it entails the existence or nonexistence of solitary waves in weakly stratified fluids. The validity of the Boussinesq approximation and the significance of the wellknown exactly linear cases are also discussed.
\end{abstract}

\section{INTRODUCTION}

Waves of permanent form progressing in a stratified fluid otherwise at rest can be studied most conveniently by adopting a frame of reference moving with the waves and thereby making the flow independent of time. The equation governing steady two-dimensional flows of a continuously stratified incompressible fluid was given in 1932 by Dubreil-Jacotin ${ }^{1}$ and independently by long. ${ }^{2}$ If Cartesian coordinates $x$ and $y$ are used, and if $\rho$ denotes the density and $\psi$ denotes the stream function, this equation is

$$
\psi_{x x}+\psi_{v y}+\frac{1}{\rho} \frac{d \rho}{d \psi}\left(\frac{\psi_{x}^{2}+\psi_{y}^{2}}{2}+g y\right)=h(\psi)
$$

where $g$ is the gravitational acceleration and

$$
h(\psi)=\frac{1}{\rho} H(\psi), \quad H \equiv p+\frac{\rho}{2} q^{2}+\rho g y,
$$

with $p$ denoting the pressure and $q$ denoting the speed. It is well known that both $\rho$ and $H$ are functions of $\psi$ alone if the flow is steady and viscous and diffusive effects are neglected. Long was able to find a set of upstream conditions for which (1) is exactly linear, and he successfully used the linear equation to study finite-amplitude waves in the lee of two-dimensional obstacles in the flow. His solution was subsequently elaborated by other workers.

The equation of Dubreil-Jacotin and Long was considerably simplified by $\mathrm{Yih}^{3}$ who used the transformation

$$
\psi^{\prime}=\int\left(\frac{\rho}{\rho_{0}}\right)^{1 / 2} d \psi
$$

$\rho_{0}$ being a constant reference density, and obtained the equation

$$
\psi_{x x}{ }^{\prime}+\psi_{y y^{\prime}}+\frac{g y}{\rho_{0}} \frac{d \rho}{d \psi^{\prime}}=h\left(\psi^{\prime}\right),
$$

in which

$$
h\left(\psi^{\prime}\right)=\frac{1}{\rho_{0}} \frac{d H}{d \psi^{\prime}} .
$$

It is immediately obvious that all the linear cases of (1) and of (3) can be obtained by simply letting $d \rho / d \psi^{\prime}$ and $h\left(\psi^{\prime}\right)$ be linear functions of $\psi^{\prime}$. Soon after the paper ${ }^{3}$ bearing (3) was presented, Long ${ }^{4}$ gave a set of upstream conditions for which (1) is exactly linear. This linear form is different from the linear form ${ }^{2}$ which he had found previously, but is one of the linear forms readily obtained from (3) and discussed in some detail by $\mathrm{Yih}^{5}$ sometime later.

Whenever (1) or (3) is exactly linear and admits solutions representing waves, such solutions are for waves of any amplitude in a continuously stratified fluid. However, there is some artificiality associated with the upstream conditions permitting (1) or (3) to be exactly linear: There is always a nonuniform velocity distribution of a parallel flow to which the solutions for waves are superposed, and this nonuniformity is always there no matter how small the amplitude of the waves. In other words, a current of nonuniform velocity must always be present for (1) or (3) to be exactly linear. For waves propagating in an otherwise quiescent fluid, it is well known that the solution for the flow in the frame of reference moving with the waves must consist of a flow with uniform velocity and a flow representing the waves, provided the amplitude of the waves is very small. Consequently, the exactly linear cases cannot correspond to progressive infinitesimal waves, and by extension cannot correspond to waves of finite amplitude (which are adjacent to infinitesimal waves) progressing in an otherwise quiescent fluid.

In this paper we wish to investigate waves of permanent form progressing in an otherwise quiescent fluid. Since the differential equation (1) or (3) is bound to be nonlinear for such waves, there is no advantage in using (3). The problem then is first to determine the functions $d \rho / d \psi$ and $h(\psi)$ in (1). This is now not as simple a task as in the study of lee waves, since the waves extend from minus infinity to infinity in the $x$ direction, and there is no parallel flow far upstream. We shall use a series expansion in powers of the amplitude, and show how the differential equation can be determined at each stage of the approximation, as well as the eigenfunctions and eigenvalues (for the wave velocity). In arriving at the mathematical solution of the problem, we are also able to assess the degree of validity of the Boussinesq approximation, which has been used so very extensively. Our solution is for any wavenumber, any stratification, and any mode of the internal wave for a given wave number. (The precise meaning of the density 
stratification will be discussed in the following section. The need for such a discussion arises from the lack of a section far upstream where the flow is parallel.) Special attention will be given to the important and realistic cases of weak density gradients.

Before formulating and solving our problem in the following section we note that this problem has already been studied by Thorpe $^{6}$ in an extensive paper. However, in Thorpe's work the Boussinesq approximation has been used and the effect of amplitude on the wave velocity has not been determined, and indeed there is no indication how that effect can be determined. Furthermore, in all previous work on waves in a continuously stratified fluid, Thorpe's included, it has never been pointed out that it is necessary to determine or specify the density distribution in the fluid when it is allowed to quiet down, so that we know for what fluid the problem is solved. This will be an important aspect of our solution to be presented in the next section.

We note that the method of determining the eigenvalue of the wave velocity at each stage of the approximation involves the requirement that the inhomogeneous part $g(y, \Delta \lambda)$ of the equation

$$
L_{\lambda} f(y)=g(y, \Delta \lambda),
$$

be orthogonal to the eigenfunction $f_{0}(y)$ satisfying

$$
L_{\lambda} f_{0}(y)=0
$$

and the boundary conditions, $L_{\lambda}$ being a linear operator containing the eigenvalue $\lambda$, and $\Delta \lambda$ being the correction to $\lambda$ that is necessary at any particular stage of the approximation under consideration. This technique was used by $\mathrm{Yih}^{7}$ to study the stability of film flow, is indispensible here, and is, in general, a powerful tool in the study of linear eigenvalue problems.

\section{FORMULATION}

The problem is to study the flow due to waves progressing in a continuously stratified fluid otherwise at rest. The fluid is specified by its density distribution when waves are absent, and this same fluid is always under consideration when waves are present.

The first task is to specify the general form of (1) that applies to our problem, whatever the density distribution of the fluid. To this end we recognize that that form must satisfy the following requirements: When the amplitude of the waves is very small, it must reduce to the well-known linear equation (for the moving frame of reference) governing infinitesimal waves, and as a consequence it must allow a parallel flow of uniform velocity (equal to the wave velocity) when the amplitude of the waves is reduced to zero. Keeping this velocity-distribution requirement in mind we see that the form sought is

$$
\psi_{x x}+\psi_{\nu y}+\frac{1}{\rho} \frac{d \rho}{d \psi}\left(\frac{\psi_{x}^{2}+\psi_{y}^{2}}{2}+g y\right)=\frac{1}{\rho} \frac{d \rho}{d \psi}\left(\frac{g}{c} \psi+\frac{c^{2}}{2}\right)
$$

where $c$ is the wave velocity. Indeed, this equation has already been given by Davis and Acrivos. ${ }^{8} \mathrm{~A}$ flow of uniform velocity $c$ in the direction of increasing $x$ has been superposed on waves progressing in the opposite direction to make the flow steady. Note that

$$
\psi=c y
$$

is always a possible solution, with no waves present at all, and that when the amplitude is small (5) indeed reduces to the wave equation for infinitesimal waves, upon replacing $\psi$ in $d \rho / d \psi$ by $c y$. Note, however, that $d \rho / d \psi$ for finite-amplitude waves is not known and cannot be specified $a$ priori. Indeed, it has to be determined and re-determined at succeeding stages of approximation, with the density-distribution requirement in mind.

We shall consider two-dimensional internal waves bounded by the horizontal boundaries $y=0$ and $y=d$, so that

$$
\psi=a \text { const at } y=0 \text { and } y=d
$$

Equations (5) and (7) constitute the eigenvalue problem, with $c$ as the eigenvalue and $\psi$ as the eigenfunction. Note that we do not demand that $\psi$ be zero at $y=0$ or at $y=d$.

It will be convenient to use the following dimensionless variables:

$$
\Psi=\psi / c d, \quad \xi=x / d, \quad \eta=y / d, \quad F^{2}=c^{2} / g d, \quad \hat{\rho}=\rho / \rho_{0},
$$

where $\rho_{0}$ is the density at $\eta=0$, and $F$ is the Froude number. In terms of these dimensionless variables, Eq. (5) becomes, after the circumflex on $\hat{\rho}$ has been dropped,

$$
\begin{gathered}
\Psi_{\xi \xi}+\Psi_{\eta \eta}+\frac{1}{\rho} \frac{d \rho}{d \Psi}\left(\frac{\Psi_{\xi}^{2}+\Psi_{\eta}^{2}}{2}+F^{-2} \eta\right) \\
=\frac{1}{\rho} \frac{d \rho}{d \Psi}\left(F^{-2} \Psi+\frac{1}{2}\right) .
\end{gathered}
$$

The boundary conditions become

$$
\Psi=\text { const } \quad \text { at } \eta=0 \text { and at } \eta=1 .
$$

In what follows we shall consider a fluid which, when at rest, has the dimensionless-density distribution

$$
\rho=\exp (-\beta \bar{\eta})
$$

where $\bar{\eta}$ is $\eta$ when no waves are present. The theory developed for the particular distribution applies to any general density distribution when the necessary changes are made to account for the density distribution specified. The "densimetric" or "internal" Froude number $F_{i}$ is defined by

$$
\beta F_{i}^{2}=F^{2}
$$

\section{THE SOLUTION}

We shall expand $\Psi$ and $F_{i}{ }^{2}$ in the series ( $A=$ amplitude)

$$
\begin{aligned}
& \Psi=\Psi_{0}+A \Psi_{1}+A^{2} \Psi_{2}+\cdots \\
& F_{i}^{-2}=G_{0}+A G_{1}+A^{2} G_{2}+\cdots
\end{aligned}
$$


It is obvious that

$$
\Psi_{0}=\eta
$$

which satisfies (9) and (11) exactly. At this stage of approximation $\eta$ is $\bar{\eta}$. Hence, from Eqs. (11) and (14) we have

$$
\frac{1}{\rho} \frac{d \rho}{d \Psi}=\frac{1}{\rho} \frac{d \rho}{d \Psi_{0}}=-\beta
$$

and (9) becomes, upon collecting terms of the power $A$,

$$
\left(\Psi_{1}\right)_{\xi \xi}+\left(\Psi_{1}\right)_{\eta \eta}-\beta\left(\Psi_{1}\right)_{\eta}=-G_{0} \Psi_{1},
$$

which is the equation governing infinitesimal waves. The solution of (16) with the boundary conditions (10), in which $\Psi_{1}$ is used for $\Psi$, gives

$$
\Psi_{1}=\exp (\beta \eta / 2) \sin n \pi \eta \sin k \xi
$$

and

$$
G_{0}=n^{2} \pi^{2}+k^{2}+\left(\beta^{2} / 4\right),
$$

where $k$ is the wavenumber and $n$ is an integer indicating the mode. For any given $k$ there are infinitely many modes corresponding to positive integral values of $n$. The larger $n$ is, the "higher" the mode.

At this stage it is necessary to re-evaluate $d \rho / d \Psi$, to see whether a new evaluation should be used for the next approximation. For this purpose we take two terms on the right-hand side of (13a), and rewrite it as

$$
\eta=\Psi-A \exp (\beta \eta / 2) \sin n \pi \eta \sin k \xi .
$$

Upon successive iterations we obtain, from (19), the result

$$
\begin{aligned}
\eta= & \Psi-A \exp (\beta \Psi / 2) \sin n \pi \Psi \sin k \xi \\
& +\frac{1}{4} A^{2} \exp (\beta \Psi)\left(n \pi \sin 2 n \pi \Psi+\beta \sin ^{2} n \pi \Psi\right) \\
& \times(1-\cos 2 k \xi)+O\left(A^{3}\right),
\end{aligned}
$$

which, upon averaging with respect to $\xi$, gives

$$
\begin{aligned}
\bar{\eta}= & \Psi+\frac{1}{8} A^{2} \exp (\beta \Psi)(\beta-\beta \cos 2 n \pi \Psi+2 n \pi \\
& \times \sin 2 n \pi \Psi)+O\left(A^{4}\right),
\end{aligned}
$$

because the average value of the terms of $O\left(A^{3}\right)$ is zero. Note that the averaging is not an inexact process, for the definition of $\bar{\eta}$ demands exactly such an averaging process.

If we write

$$
\frac{1}{\rho} \frac{d \rho}{d \Psi}=\frac{1}{\rho} \frac{d \rho}{d \tilde{\eta}} \frac{d \bar{\eta}}{d \Psi}=-\beta \frac{d \bar{\eta}}{d \Psi},
$$

and substitute this into (9), we have

$$
\begin{aligned}
\Psi_{\xi \xi} & +\Psi_{\eta \eta}-\beta \frac{d \bar{\eta}}{d \Psi}\left(\frac{\Psi_{\xi}{ }^{2}+\Psi_{\eta}{ }^{2}}{2}+F^{-2} \eta\right) \\
= & -\beta \frac{d \bar{\eta}}{d \Psi}\left(F^{-2} \Psi+\frac{1}{2}\right) .
\end{aligned}
$$

Using (21) in (22), we find that the terms of $O\left(A^{2}\right)$ in Eq. (21) introduce only terms of $O\left(A^{3}\right)$ in Eq. (22). Therefore, while we are determining the terms of $O\left(A^{2}\right)$ in $\Psi$, we need not yet make the correction for $d \rho / d \Psi$.

\section{Keeping}

$$
\frac{2}{\rho} \frac{d \rho}{d \Psi}=-\beta
$$

then, we can proceed with the determination of $\Psi_{2}$. Using Eqs. (13a), (13b), and (14) and gathering terms of $O\left(A^{2}\right)$ in (9), we obtain

$$
\nabla^{2} \Psi_{2}-\beta\left(\Psi_{2}\right)_{\eta}+G_{0} \Psi_{2}=\beta S-G_{1} \Psi_{1},
$$

where

$$
\nabla^{2}=\frac{\partial^{2}}{\partial \xi^{2}}+\frac{\partial^{2}}{\partial \eta^{2}}
$$

and, after some reductions,

$$
S=\exp (\beta \eta)\left(B_{1}+B_{2} \cos 2 n \pi \eta+B_{3} \sin 2 n \pi \eta\right),
$$

where

$$
8 B_{1}=G_{0}, \quad 32 B_{2}=4 n^{2} \pi^{2}-4 k^{2}-\beta^{2}, \quad 8 B_{3}=n \pi \beta .
$$

Solving (23) with the boundary conditions (10), we find that

$$
G_{1}=0,
$$

and

$$
\begin{aligned}
\Psi_{2}= & \beta\left\{B_{1} f_{1}(\eta)+B_{2} f_{2}(\eta)+B_{3} f_{3}(\eta)\right. \\
& \left.-\left[B_{1} f_{4}(\eta)+B_{2} f_{5}(\eta)+B_{3} f_{6}(\eta)\right] \cos 2 k \xi\right\},
\end{aligned}
$$

where

$$
\begin{aligned}
f_{1}(\eta)= & G_{0}^{-1} \exp (\beta \eta), \\
f_{2}(\eta)= & (1 / M) \exp (\beta \eta)\left[\left(G_{0}-4 n^{2} \pi^{2}\right)\right. \\
& \times \cos 2 n \pi \eta+2 n \pi \beta \sin 2 n \pi \eta], \\
f_{3}(\eta)= & (1 / M) \exp (\beta \eta)\left[\left(G_{0}-4 n^{2} \pi^{2}\right)\right. \\
& \times \sin 2 n \pi \eta-2 n \pi \beta \cos 2 n \pi \eta], \\
f_{4}(\eta)= & \left(G_{0}-4 k^{2}\right)^{-1} \exp (\beta \eta / 2)[\exp (\beta \eta / 2) \\
& -\cos \gamma \eta-a \sin \gamma \eta], \\
f_{5}(\eta)= & (1 / N) \exp (\beta \eta / 2)\{-P[\exp (\beta \eta / 2) \cos 2 n \pi \eta \\
& -\cos \gamma \eta-a \sin \gamma \eta]+2 n \pi \beta \exp (\beta \eta / 2) \sin 2 n \pi \eta\}, \\
f_{6}(\eta)= & (1 / N) \exp (\beta \eta / 2)\{-P \exp (\beta \eta / 2) \sin 2 n \pi \eta \\
& -2 n \pi \beta[\exp (\beta \eta / 2) \cos 2 n \pi \eta-\cos \gamma \eta-a \sin \gamma \eta]\},
\end{aligned}
$$

with

$$
\begin{aligned}
& M=\left(G_{0}-4 n^{2} \pi^{2}\right)^{2}+4 n^{2} \pi^{2} \beta^{2}, \quad P=4 n^{2} \pi^{2}+4 k^{2}-G_{0} \\
& N=P^{2}+4 n^{2} \pi^{2} \beta^{2}
\end{aligned}
$$


and

$$
\begin{aligned}
& \gamma=\left(G_{0}-4 k^{2}-\frac{\beta^{2}}{4}\right)^{1 / 2} \\
& a=(\exp (\beta / 2)-\cos \gamma)(\sin \gamma)^{-1}
\end{aligned}
$$

The result (26) is obtained because the term $\beta S$ does not contain the factor $\sin k \xi$ or $\cos k \xi$, and if $G_{\mathbf{1}}$ did not vanish there would be no solution for $\Psi_{2}$. This has already been mentioned in Sec. I, in connection with (4). Indeed, since $\Psi_{1}$ contains $\sin k \xi$, the particular solution of $(23)$ to account for the term $-G_{1} \Psi_{1}$ must also contain it. Then, denoting that particular solution by $p_{1}(\eta) \sin k \xi$, we have $p_{1}(0)=0=p_{1}(1)$ and

$$
L p_{1}=-G_{1} \exp (\beta \eta / 2) \sin n \pi \eta,
$$

where

$$
L=\frac{d^{2}}{d \eta^{2}}-\beta \frac{d}{d \eta}+G_{0}-k^{2} .
$$

On the other hand, (16) can be written as

$$
L \exp (\beta \eta / 2) \sin n \pi \eta=0 .
$$

Multiplying (31) by $\exp (-\beta \eta / 2) \sin n \pi \eta$ and (33) by $\exp (-\beta \eta) p_{1}$, integrating between zero and 1 , using the boundary conditions for $p_{1}$, and taking the difference of the two integrated equations, we have (26).

The functions $f_{4}, f_{5}$, and $f_{6}$ need a discussion because the $\gamma$ defined in (30) may be zero or equal to $m \pi$, with $m$ equal to an integer and less than $n$. In either case $\sin \gamma$ vanishes and the number $a$ in (30) is infinite. The case $\gamma=0$ is not really troublesome because the complimentary solutions $\exp (\beta \eta / 2) \cos \gamma \eta$ and $\exp (\beta \eta / 2) \sin \gamma \eta$ in $f_{4}, f_{5}$, and $f_{6}$ can be replaced by $\exp (\beta \eta / 2)$ and $\eta \exp (\beta \eta / 2)$, respectively, and with the constant $a$ replaced by $\exp (\beta / 2)-1$. The case $\gamma=m \pi$, with $m=$ a nonzero integer less than $n$ is much more significant. In this case

$$
G_{0}=n^{2} \pi^{2}+k^{2}+\frac{\beta^{2}}{4}=m^{2} \pi^{2}+4 k^{2}+\frac{\beta^{2}}{4}
$$

and a look at (16) shows that whenever (34) holds the $n$th mode of internal waves with wavenumber $k$ has the same wave velocity as the $m$ th mode with wavenumber $2 k$. In such a case it is necessary (in order to have internal waves of permanent form) to have first-order solutions in addition to $\Psi_{1}$ given by (17). These are solutions of (16) but with wavenumbers zero and $2 k$, respectively, and are

$$
\begin{aligned}
& \chi_{1}=A \exp (\beta \eta / 2)\left(a_{1} \sin \mu \eta+b_{1} \cos \mu \eta\right), \\
& \mu=\left(G_{0}-\frac{\beta^{2}}{4}\right)^{1 / 2}, \\
& \chi_{2}=A a_{2} \exp (\beta \eta / 2) \sin m \pi \eta \cos 2 k \xi,
\end{aligned}
$$

in which $a_{2}$ is arbitrary. This means we can start with any two wave amplitudes for the wave trains of wavenumbers $k$ and $2 k$. When (35) and (36) are included in $\Psi_{1}$ in (13a) and (13a) is substituted into (22), terms of $O\left(A^{2}\right)$ are collected, and then terms with factors $\sin k \xi$ and $\cos 2 k \xi$ are separated, we obtain two equations:

$$
L \theta_{1}=a_{1} g_{1}(\eta)+b_{1} h_{1}(\eta)+a_{2} g_{2}(\eta),
$$

where $L$ is the operator defined by (32), and

$$
\begin{aligned}
\left(\frac{d^{2}}{d \eta^{2}}-\beta \frac{d}{d \eta}+G_{0}-4 k^{2}\right) \theta_{2}= & g_{0}(\eta)+a_{1} a_{2} g_{3}(\eta) \\
& +b_{1} a_{2} g_{4}(\eta) .
\end{aligned}
$$

By making the right-hand sides of Eqs. (37) and (38) orthogonal to $\exp (\beta \eta / 2) \sin n \pi \eta$ and $\exp (\beta \eta / 2) \sin m \pi \eta$, respectively, we can determine $a_{1}$ and $b_{1}$. The solution $\theta_{2}$ then replaces the coefficient of $\cos 2 k \xi$ in (27) and the solution $\theta_{1} \sin k \xi$ is the additional part which is now necessary.

Note that to $f_{1}, f_{2}$, and $f_{3}$ in (28) could be added solutions of the equation

$$
\left(\frac{d^{2}}{d \eta^{2}}-\beta \frac{d}{d \eta}+G_{0}\right) \theta_{11}=0 .
$$

This is the reason that Thorpe $^{6}$ decided that parallel flows (currents), which can be added to the flow sought by him, can be determined only if one knows how the waves have been created. Actually, there does not appear to be any hope that these currents can really be determined from the circumstances of generation of the waves. Rather, the purpose is to isolate waves of fundamental wavenumber $k$ as much as possible, avoiding adding any terms not having this wavenumber at all stages of approximation unless addition of such terms is necessary as particular solutions at higher approximations than the first, or even at the first approximation, as in the case $\gamma=m \pi$ discussed in the preceding paragraph. With this decision, the parallel currents are no longer arbitrary, but uniquely determined, except in the case $\gamma=m \pi$ discussed above, where $a_{2}$ is arbitrary and hence also $a_{1}$ and $b_{1}$. That exceptional case arises because waves of wavenumbers $k$ and $2 k$ are no longer isolatable at the order $O(A)$.

One more point must be clarified before we go on to deal with terms of $O\left(A^{3}\right)$. If $\gamma$ is not equal to $m \pi$ exactly but very near to it, the value of $a$ in (30) can be very large, particularly if $m$ is even. Then, $\Psi_{2}$ can be very large except for certain values of $\eta$, indeed very much larger than $\Psi_{1}$, and this seems strange and unreasonable. The apparent difficulty is removed when we remember that in the next stage of approximation large contributions are fed back in the same way to the term containing the factor $\sin k \xi$, and the amplitude will not be $A$ but redetermined. Then, the ratio of $\Psi_{2}$ to $\Psi_{1}$ will not be so large. Difficulties of the kind discussed in this and the preceding paragraphs are typical of nonlinear problems. Their resolutions are never immediately clear and often, as in this case, require a great deal of thought. Note that if $n=1$ (for the first mode), then nonzero value of $m$ does not exist, and the apparent difficulties discussed in the preceding paragraphs do not exist. For $n=1$, however, there is still the possibility that the $M$ defined in (29), which never vanishes, may nevertheless be of $O\left(\beta^{2}\right)$, thus making $f_{2}$ and $f_{3}$ in (28) very large. For the convenience of later discussions, we shall only deal with those values of $k^{2}$ which satisfy

$$
3 \pi^{2}-k^{2}=O(1)
$$


for $n=1$. For higher values of $n$, to avoid the case in which $\gamma$ is equal or near $m \pi$, we shall assume that Eq. (34) is never exactly or nearly satisfied, or that, with $n=2$ and $m=1$ to obtain the safe bound for $k^{2}$,

$$
\pi^{2}-k^{2}=O(1)
$$

If (40b) is satisfied, then $M$ defined in (29) is not small. Hence for $n>1$, we impose (40b) only. Since we have carried out our calculations only to $O\left(A^{2}\right)$ in $(13 \mathrm{a}, \mathrm{b})$, our conclusions will necessarily be valid only to this order. This imposes a limit (undetermined) on the magnitude of $|A|$.

Before going on with our calculation, it is now necessary to recalculate $\rho^{-1} d \rho / d \Psi$. Using (13a) and (27), we obtain

$$
\begin{aligned}
\bar{\eta}= & \Psi+\frac{1}{8} A^{2} \exp (\beta \Psi)(\beta-\beta \cos 2 n \pi \Psi+2 n \pi \Psi \sin 2 n \pi \Psi) \\
& +\beta A^{2}\left[B_{1} f_{1}(\Psi)+B_{2} f_{2}(\Psi)+B_{3} f_{3}(\Psi)\right]+O\left(A^{4}\right)
\end{aligned}
$$

Substituting (41) into (22); using (13), and collecting terms of $O\left(A^{3}\right)$, we have

$$
\left(\nabla^{2}-\beta \frac{\partial}{\partial \eta}+G_{0}\right) \Psi_{3}=T_{1}+T_{2}+T_{3}-G_{2} \Psi_{1}
$$

where

$$
\begin{aligned}
T_{1}= & \beta^{2} \phi^{\prime}\left[B_{1} f_{1}^{\prime}+B_{2} f_{2}^{\prime}+B_{3} f_{3}^{\prime}\right. \\
& \left.+0.5\left(B_{1} f_{4}^{\prime}+B_{2} f_{5}^{\prime}+B_{3} f_{6}^{\prime}\right)(\sin k \xi-\sin 3 k \xi)\right] \\
& +k^{2} \beta^{2} \phi\left(B_{1} f_{4}+B_{2} f_{5}+B_{3} f_{6}\right)(\sin 3 k \xi-\sin k \xi) \\
8 T_{2}= & \left(\beta \phi^{\prime}-G_{0} \phi\right)[\exp (\beta \eta)(\beta-\beta \cos 2 n \pi \eta \\
& +2 n \pi \sin 2 n \pi \eta)]^{\prime} \\
T_{3}= & \beta\left(\beta \phi^{\prime}-G_{0} \phi\right)\left(B_{1} f_{1}^{\prime}+B_{2} f_{2}{ }^{\prime}+B_{3} f_{3}^{\prime}\right)
\end{aligned}
$$

with

$$
\phi=\exp (\beta \eta / 2) \sin n \pi \eta
$$

The accents in (43) indicate differentiation with respect to $\eta$, and the functions $f_{1}$ to $f_{6}$ are functions of $\eta$.

To determine $G_{2}$, we again demand that the right-hand side of (42) be orthogonal to $\Psi_{1}$, that is to say, the sum $J$ of the coefficients of $\sin k \xi$ of the right-hand side of $(42)$ be orthogonal to $\phi$ in the sense that

$$
\int_{0}^{1} \exp (-\beta \eta) J \phi d \eta=0
$$

After we determine $G_{2}$, we can find $\Psi_{3}$ by solving (42). We shall determine $G_{2}$ to see how the amplitude $A$ affects the wave velocity, but we shall not attempt to determine $\Psi_{3}$. That determination is straightforward, but tedious.

Note that $T_{1}$ arises from the term containing $|\nabla \Psi|^{2}$ in (22), $T_{2}$ and $T_{3}$ arise from the displacement of isopycnic lines as the result of $\Psi_{1}$ and $\Psi_{2}$, respectively. For all cases important in practice, $\beta$ is very small; therefore, we shall consider $\beta$ to be small. This will simplify the presentation of the results, although $G_{2}$ can be determined from (45) completely, with all terms included, in a straightforward although lengthy way. For small $\beta$, we shall show that the dominant term in $G_{2}$ comes from $T_{2}$.

If (40) is satisfied for $n=1$ and (40b) satisfied for $n>1$, it can be shown that all the contributions to $G_{2}$ from $T_{1}$ (or rather those terms of $T_{1}$ that contain $\sin k \xi$ ) are $O\left(\beta^{2}\right)$. The same is true of the contributions from $T_{3}$. The details supporting these statements can be obtained in a straightforward manner, but are very lengthy. We shall omit their presentation to save space.

Carrying out in full the contributions of $T_{2}$, we find that

$$
\begin{aligned}
& \int_{0}^{1} \exp (-\beta \eta) T_{2} \phi d \eta \\
&= 0.5 \beta^{4} I(\beta, n \pi)+\beta^{2}\left(n^{2} \pi^{2}-0.25 \beta^{2}\right)[I(\beta, 3 n \pi) \\
&-I(\beta, n \pi)]+n \pi \beta^{3}[R(\beta, n \pi)-R(\beta, 3 n \pi)] \\
&+0.5 n \pi \beta\left\{\beta^{2} I(\beta, 2 n \pi)+\left(2 n^{2} \pi^{2}-0.5 \beta^{2}\right) I(\beta, 4 n \pi)\right. \\
&+2 n \pi \beta[R(\beta, 0)-R(\beta, 4 n \pi)]\} \\
&-\frac{1}{2} G_{0}\left\{\beta^{2} R(\beta, 0)+\left(4 n^{2} \pi^{2}-2 \beta^{2}\right) R(\beta, 2 n \pi)\right. \\
&+4 n \pi \beta I(\beta, 2 n \pi)-2 n \pi \beta I(\beta, 4 n \pi) \\
&\left.+\left(0.5 \beta^{2}-2 n^{2} \pi^{2}\right)[R(\beta, 0)+R(\beta, 4 n \pi)]\right\},
\end{aligned}
$$

in which the functions $R$ and $I$ are defined by

$$
\begin{aligned}
& R(p, q)+i I(p, q)=\int_{0}^{1} \exp [(p+i q) \eta] d \eta \\
& =\frac{1}{p^{2}+q^{2}}[p(\exp (p) \cos q-1)+q \exp (p) \sin q] \\
& \quad+\frac{i}{p^{2}+q^{2}}[p \exp (p) \sin q-q(\exp (p) \cos q-1)] .
\end{aligned}
$$

If $q=r \pi$ ( $r$ is an integer $)$,

$$
\begin{aligned}
& R(p, r \pi)=\frac{p}{p^{2}+r^{2} \pi^{2}}\left[\exp (p)(-1)^{r}-1\right], \\
& I(p, r \pi)=\frac{r \pi}{p^{2}+r^{2} \pi^{2}}\left[\exp (p)(-1)^{r}-1\right] .
\end{aligned}
$$

Thus

$$
R(\beta, 0)=1+0.5 \beta+O\left(\beta^{2}\right)
$$

and, with $s$ equal to any positive integer,

$$
\begin{aligned}
& R(\beta, 2 s-1)=O(\beta), \quad R(\beta, 2 s)=O\left(\beta^{2}\right), \\
& I(\beta, 2 s-1)=O(1), \quad I(\beta, 2 s)=O(\beta) .
\end{aligned}
$$

The right-hand side of (46) is therefore equal to

$$
n^{2} \pi^{2} G_{0}(1+0.5 \beta)+O\left(\beta^{2}\right)
$$

and, applying (45) and remembering the restrictions (40a) and (40b),

$$
G_{2}=n^{2} \pi^{2} G_{0}(2+\beta)+O\left(\beta^{2}\right) .
$$


That $G_{2}$ is positive means that $F_{i}^{-2}$ increases with $A^{2}$, according to $(13 \mathrm{~b})$, or that $c^{2}$ decreases with $A^{2}$. This is a rather unexpected result, since we are so used to the increase of wave velocity with amplitude for progressive gravity waves, as for the well-known Stokesian waves. We note, however, that already for gravity waves in two superposed layers of homogeneous liquids $\mathrm{Hunt}^{9}$ and Thorpe $^{6}$ (who made some corrections of Hunt's work) have shown that it is possible for $c^{2}$ to decrease with amplitude. (In their case it happens for long waves and small density differences.) The result (48) is remarkable in that for a continuously stratified fluid $c^{2}$ always decreases with the amplitude. Perhaps this result needs some interpretation. In this writer's opinion, it happens because a larger amplitude in a wave motion "squeezes" the isopycnic lines against the upper solid boundary near the crests, thereby increasing the vertical density gradient near the upper solid boundary and decreasing the density gradient below. Similarly, the wave motion also "squeezes" the isopycnic lines against the lower solid boundary near the troughs, increasing the density gradient there and decreasing it above. Since the increase of density gradient near solid boundaries are quite ineffective in increasing $c^{2}$, whereas the decrease of it in a wider region is more effective in decreasing it, $c^{2}$ decreases with the amplitude.

We have specified the stratification of $\rho$ to be exponential by (11). However, the conclusions concerning the decrease of $c^{2}$ with $A^{2}$ for small $\beta$ will remain valid for any stratification for which

$$
\frac{1}{\rho} \frac{d \rho}{d \bar{\eta}}=\beta+O\left(\beta^{2}\right), \beta \ll 1 .
$$

In particular, they remain valid for the important case of a linear stratification, for which

$$
\frac{d \rho}{d \bar{\eta}}=\text { const. }
$$

Whether $\beta$ is small or not, and for any stratification, the method of solution presented here applies to finite-amplitude waves in any stratified fluid.

Before we conclude this section, we note that the averaging process leading to (21) is valid only if there are no regions of closed streamlines. This requirement places a restriction on the admissible values of $A^{2}$. However, if $A^{2}$ is so large that regions of closed streamlines do occur, the modification required is obvious: All we have to do is to find how much fluid there is between any two streamlines (or isopycnic lines). The only thing that has to be kept in mind is that the solution must be such that when the fluid is allowed to quiet down it will have the stratification we specify for it.

\section{THE EXISTENCE OF INTERNAL SOLITARY WAVES}

Solitary waves are classified as long waves, and they invariably owe their existence to the crucial requirement that the "local" wave velocity at the point of greatest vertical displacement be greater than the wave velocity of infinitesimal long waves. For a layer of homogeneous liquid, this requirement is satisfied (Ref. 10, p. 424). For two semi-infinite homogeneous liquids with an interface, Hunt ${ }^{9}$ has shown that this requirement is met. (See Ref. 6, p. 570.) For two homogeneous liquids of equal, finite depths bounded above and below by solid boundaries, whether this requirement is met or not depends on the relative magnitudes of the wave length and the density difference [Hunt, ${ }^{9}$ as corrected by Thorpe, ${ }^{6}$ p. 571, Eq. (2.1.6) ]. This fact agrees with the conclusion of Long. ${ }^{11}$

Solitary waves in a continuously stratified fluid of finite depth, bounded above and below by horizontal boundaries have been treated elegantly by Benjamin ${ }^{12}$ and ingeniously by Long. ${ }^{10}$ In both papers it was assumed, for the existence of solitary waves, that the velocity of finite-amplitude waves increases with amplitude. See Benjamin ${ }^{12}$ (p. 243, first paragraph) and Long ${ }^{11}$ [Eqs. (18), (42), and (66)]. Neither author has inquired whether this assumption can be met, and their treatments based on this assumption are indeed correct. [In (4.21) of Benjamin's paper, $c^{2}$ ( $c$ is the velocity of solitary waves) is shown to be greater than ${c_{n}}^{2}$ $\left(c_{n}\right.$ is the velocity of corresponding long waves of infinitesimal amplitude) on the assumption that his $\Delta$, or $\epsilon J$, or $\gamma_{n}$, is positive. All this is very consistent, but $\gamma_{n}$ has not been independently shown to be positive.] Yet, it is difficult to escape the impression that they never doubted the truth of this assumption. The conclusion we have reached in the preceding paragraph shows that this crucial assumption is indeed not met for one continuously and weakly stratified fluid of finite depth between two solid boundaries, and strongly indicates that solitary waves, in such a fluid, as illustrated in Fig. 2 of Thorpe's paper, ${ }^{6}$ may not exist. Note that we have expanded the eigenfunction and the eigenvalue in powers of the amplitude $A$, with $\beta$ and $k$ appearing in the coefficients of this expansion in a complete and indeed closed manner, so that the treatment here is somewhat more accurate and reliable than an expansion in $A, \beta$, and $k$ concurrently, as was done by Long. ${ }^{11}$

All this is not to say that solitary waves do not exist if the density is discontinuous, or if, although continuous, extend to infinity. Indeed, Davis and Acrivos ${ }^{8}$ have shown experimentally that solitary waves exist in a stratified fluid layer imbedded between two homogeneous layers of great depths (in comparison to the stratified layer), of densities $\rho_{1}$ and $\rho_{2}$, and thus vindicating their own theory as well as Benjamin's ${ }^{13}$ for such a fluid system. Since infinite extensions are unrealistic, and the experiments of Davis and Acrivos were necessarily for finite depths, a reconciliation of this fact with the implication of the results in Sec. III is necessary. The author believes that the explanation lies in the importance of the magnitude of the wavenumber $k$. If the length scale is that of the stratified layer in Davis and Acrivos' experiment, waves that are long with respect to that scale may be short with respect to the total depth. Since in our study the wavelength is measured in terms of the total depth, our wavenumber may be very large for waves that are long with respect to the stratified layer, and therefore even for weak stratifications terms of order $O\left(\beta^{2}\right)$, which are neglected in reaching the conclusion that wave velocity decreases with the amplitude, may not be negligible because the coefficients are high. This seems to indicate that the existence or nonexistence of solitary waves in a continuously stratified fluid not only depends on the weakness of the stratification, but also on the distribution of stratification. If the density gradient is nearly uniform throughout in the sense of (49), solitary waves may not exist, for a calculation for any such fluid, not necessarily 
exponentially stratified, will lead to the same conclusion that we reached in Sec. III.

We do not, however, wish to be very dogmatic about our doubt of the nonexistence of solitary waves between fixed boundaries even for a fluid satisfying (49), for the link between progressive periodic waves and solitary waves is only the tenuous one of long wave length. This may be too tenuous a link on which to base any strong conclusions. For instance, in the calculation of $\bar{\eta}$ in terms of $\psi$ in (21), the periodicity played an important role, and solitary waves are not periodic. What this discussion is intended to point out is that to be convincing, it is desirable, in any study of solitary waves, to show independently that the speed of finite-amplitude waves, especially solitary waves, is indeed greater than the velocity of waves of infinitesimal amplitude.

\section{VALIDITY OF THE BOUSSINESQ APPROXIMATION}

In the present context, the Boussinesq approximation amounts to ignoring the term $|\nabla \psi|^{2}$ and writing $\rho_{0}{ }^{-1} d \rho / d \psi$ for $\rho^{-1} d \rho / d \psi$ in (1), $\rho_{0}$ being a constant, mean density. Thus, the Boussinesq approximation does not necessarily make (1) linear. If we make the Boussinesq approximation in the present problem, $\Psi_{1}$ will be modified to the extent of a term of $O(\beta)$, and $\Psi_{2}$ will be modified. The term $G_{2}$ will be modified by an amount of at most $O(\beta)$ if $k$ satisfies (40a) or (40b). Thus, the Boussinesq approximation will not affect the value of $F_{i}^{-2}$ by an appreciable amount if $\beta$ is small and $k$ is not too large.

If we merely ignore the term $|\nabla \psi|^{2}$ in (1) and carry out the calculations for the exponentially stratified fluid, as in Sec. III, we shall find that $\Psi_{0}$ and $\Psi_{1}$ are unaffected, $\Psi_{2}$ is zero, and (48) always holds.

\section{THE EXACTLY LINEAR CASES OF (3)}

As shown by Yih, ${ }^{5}$ the exactly linear cases of (1) can be found most easily from (3), by simply setting $d \rho / d \psi^{\prime}$ and $h\left(\psi^{\prime}\right)$ to be linear functions of $\psi^{\prime}$. The solutions for (3), when it is exactly linear, can then be obtained for any amplitude of the waves. The exactly linear cases all require a parallel flow of nonuniform velocity superposed on the wave motion. Granted this requirement, it is generally accepted that waves of any mode propagate with the same velocity regardless of the amplitude, in a stratified fluid permitting (3) to be exactly linear.

This, however, would be a somewhat superficial conclusion because the distribution of density in space varies with the amplitude, as we have shown, and the density stratification, if the waves are allowed to die out, would be different for different amplitudes of the waves. Hence, when we say the wave velocity is the same for any amplitude for the exactly linear cases, we are really comparing solutions not only for different wave amplitudes but for different fluids. This much neglected fact reduces the significance of the exactly linear cases.

\section{CONCLUSIONS}

On the assumption that the procedure of solution used in this paper is convergent, from the foregoing analysis and discussion we conclude that

1. Finite-amplitude waves of permanent form in any stably stratified fluid between two horizontal boundaries exist, and there are infinitely many modes. (The stability of these waves is another question.)

2. The method of solution presented in this paper can be applied to find the eigenfunction and the wave velocity for internal waves of finite amplitude in any stratified fluid.

3) In exceptional cases described by equations like (34), it is necessary to start with more than one wavenumber even at the first approximation.

4. For the first mode, calculations up to the order $O\left(A^{2}\right)$ for the eigenfunction and for the wave velocity (contained in $F_{i}{ }^{2}$ ) show that for an exponentially stratified fluid and for a weak stratification the square of the wave velocity always decreases with the square of the amplitude provided (40a) is satisfied, so that the wavelength is not too small. This conclusion is especially accurate when the amplitude is small, and holds for other weakly stratified fluids too.

5 . Conclusion 4 is valid for higher modes also, provided (40b) holds. Otherwise, further approximations are necessary to insure sufficient accuracy.

6. As a consequence of conclusions 4 and 5 , the existence of internal solitary waves in a weakly stratified fluid between solid boundaries, whose density satisfies (49), is somewhat in doubt.

7. For weak stratifications and if the wavelengths are not very short, the Boussinesq approximation indeed gives reliable results for the wave velocity.

8. The solutions for different amplitudes for any of the exactly linear cases of (1) or (3) really correspond not to the same fluid, but to different fluids.

\section{ACKNOWLEDGMENTS}

This work has been jointly sponsored by the Office of Naval Research and the National Science Foundation.

${ }^{1}$ M. L. Dubreil-Jacotin, Atti Accad, Naz. Linei Cl. Sci. Fis. Mat. Nat. Rend. 15, 814 (1932).

${ }^{2}$ R. R. Long, Tellus 5, 42 (1953).

' C.-S. Yih, in Proceedings of the Third U.S. National Congress on Applied Mechanics (American Society of Mechanical Engineers, New York, 1958), p. 857

${ }^{4}$ R. R. Long, Q. J. R. Meteorol. Soc. 84, 159 (1958).

${ }^{5}$ C.-S. Yih, J. Fluid Mech. 9, 161 (1960).

' S. A. Thorpe, Phil. Trans. R. Soc. Lond. 263, 563 (1968).

${ }^{7}$ C.-S. Yih. Phys. Fluids 6, 321 (1963).

8 R. E. Davis and A. Acrivos, J. Fluid Mech. 29, 593 (1967).

${ }^{2} \mathrm{~J}$. N. Hunt, La Houille Blanche 4, 515. (1961).

${ }^{10} \mathrm{H}$. Lamb, Hydrodynamics (Cambridge University Press, Cambridge, 1932).

${ }^{11}$ R. R. Long, Tellus 17, 46 (1965).

12 T. B. Benjamin, J. Fluid Mech. 25, 241 (1966)

${ }^{13}$ T. B. Benjamin, J. Fluid Mech. 29, 559 (1967). 